\title{
Bile Cast Nephropathy in Patients with Acute Kidney Injury Due to Hepatorenal Syndrome: A Postmortem Kidney Biopsy Study
}

\author{
Suman Lata Nayak ${ }^{\# 1}$, Manoj Kumar ${ }^{* 2}$, Chhagan Bihari ${ }^{3}$ and Archana Rastogi ${ }^{3}$ \\ ${ }^{1}$ Department of Nephrology, Institute of Liver and Biliary Sciences, New Delhi, India; ${ }^{2}$ Department of Hepatology and Liver \\ Transplatation, Institute of Liver and Biliary Sciences, New Delhi, India; ${ }^{3}$ Department of Pathology, Institute of Liver and \\ Biliary Sciences, New Delhi, India
}

\begin{abstract}
Background and Aims: The role of bile cast nephropathy $(B C N)$ in pathogenesis of hepatorenal syndrome (HRS) in decompensated cirrhosis and acute on chronic liver failure (ACLF) is unknown. This study aimed to determine the frequency of BCN detected on postmortem renal biopsy among patients with decompensated cirrhosis and ACLF who had been admitted with acute kidney injury due to HRS (HRAAKI) and expired during that hospitalization. Methods: One-hundred-twenty-seven postmortem renal biopsies with adequate size ( $>1 \mathrm{~cm}$ in length) were included for analysis. These were obtained from 84 patients with decompensated cirrhosis and 43 patients with ACLF. Results: BCN was detected in 57 of the total $127(44.8 \%)$ renal biopsy specimens. Patients with BCN had significantly higher levels of serum total bilirubin, total leukocyte count and model for end-stage liver disease score, as compared to those without BCN. BCN was detected in $32 / 43(74.4 \%)$ of the patients with ACLF, as compared to $25 / 84(29.7 \%)$ of the patients with decompensated cirrhosis $(p<0.001)$. On multivariate analysis, direct bilirubin (OR (95\% CI): $1.27(1121-1.698) ; p<0.001)$ and presence of ACLF (OR (95\% CI): 2.603 (1.054-7.111); $p=0.041$ ) were found to be significant predictors of $\mathrm{BCN}$ on postmortem renal biopsy. Conclusion: BCN was found in $72.1 \%$ of patients with ACLF and $27.4 \%$ patients with decompensated cirrhosis who had been hospitalized with an admitting diagnosis of HRS-AKI and who expired during that hospitalization and underwent postmortem renal biopsy. Direct serum bilirubin and presence of ACLF were found to be significant predictors of BCN on postmortem renal biopsy. Citation of this article: Nayak SL, Kumar M, Bihari C, Rastogi A. Bile cast nephropathy in patients with acute kidney
\end{abstract}

\#These two authors contributed equally to this work.

Keywords: Bile cast nephropathy; Acute kidney injury; Hepatorenal syndrome. Abbreviations: AKI, acute kidney injury; ESLD, end-stage liver disease; ACLF acute on chronic liver failure; ATN, acute tubular necrosis; HRS, hepatorenal syndrome; BCN, bile cast nephropathy; HRS-AKI, acute kidney injury due to hepatorenal syndrome; RBCs, red blood cells; HPF, high power field; APASL, Asian Pacific Association for the Study of the Liver; INR, international normalization ratio of prothrombin time; ATI, acute tubular injury; GFR, glomerular filtration rate; ROC, receiver operating characteristic; MELD, model for end-stage liver disease; AUROC, area under the receiver operating characteristic.

Received: 15 November 2017; Revised: 15 March 2017; Accepted: 31 March 2017

*Correspondence to: Manoj Kumar, Department of Hepatology, Institute of Liver and Biliary Sciences, D1 Vasant Kunj, New Delhi 110070, India. Tel: +91-1146300000, Fax: +91-11-46300063, E-mail: manojkumardm@gmail.com injury due to hepatorenal syndrome: a postmortem kidney biopsy study. J Clin Transl Hepatol 2017;5(2):92-100. doi: 10.14218/JCTH.2016.00063.

\section{Introduction}

Acute kidney injury (AKI) occurs frequently in patients with end-stage liver disease (ESLD) and acute on chronic liver failure (ACLF), and portends a poor prognosis. ${ }^{1}$ AKI can be due to prerenal, intrinsic renal and postrenal factors. Prerenal injury results from renal hypoperfusion without damage to the glomerulus or tubules. Left untreated, prerenal injury can progress to acute tubular necrosis (ATN), a type of intrinsic renal injury. Of these, prerenal etiology-either volume responsive (e.g., use of diuretics, gastrointestinal bleeding, infection, diarrhea, nausea/vomiting and large volume paracentesis without albumin infusion) or volume nonresponsive (i.e. hepatorenal syndrome, HRS) - is the most common cause of AKI among patients with cirrhosis and ACLF followed by ATN; this occurrence is common either as a complication of sepsis or due to unrecognized, untreated pre-renal injury and nephrotoxic drugs, while the postrenal etiology due to urinary tract obstruction is extremely rare. ${ }^{2-4}$

Bile cast nephropathy (BCN) is AKI/renal dysfunction in patients with jaundice, and is accompanied by typical histological changes, including a broad spectrum of renal injury, predominantly tubular epithelial injury, together with intraluminal bile cast formation. ${ }^{5-7} \mathrm{BCN}$ has been described in a variety of circumstances, like obstructive jaundice, ${ }^{5,8}$ druginduced severe cholestatic liver injury, ${ }^{9}$ nonimmune hydrops fetalis-associated jaundice, ${ }^{10}$ infectious mononucleosisassociated jaundice and renal failure, ${ }^{11}$ hemolytic jaundice and cirrhosis, and fulminant hepatitis. 6,12

AKI due to HRS, known as HRS-AKI, is usually precipitated by infections/sepsis and has high mortality, with a median survival of around $50 \%$ at 2 weeks. ${ }^{13}$ HRS-AKI is a functional and potentially reversible form of renal failure, without any major structural or histological changes in the kidneys and which is characterized by intense renal vasoconstriction. ${ }^{14}$ Only about $35-50 \%$ of patients with HRS-AKI have a favorable response to albumin and terlipressin treatment. However, the role BCN plays in pathogenesis and progression of HRS-AKI remains elusive. Only one case series of BCN in patients with cirrhosis has been published to date. ${ }^{6}$ This study, consisting of 41 autopsy cases and 3 patients with 
kidney biopsies, studied cholemic nephropathy in jaundiced patients with cirrhosis $(n=23)$, cholestatic/obstructive jaundice $(n=13)$, severe acute liver dysfunction $(n=5)$ and hemolytic jaundice $(n=2)$. Among the 44 total patients, 13 were clinically classified as having HRS; however, 11 $(85 \%)$ out of these 13 showed bile casts on kidney histology. There are no studies of BCN in patients with ACLF.

The aim of this study was to determine the frequency of $\mathrm{BCN}$ and other renal biopsy findings on postmortem renal biopsy among patients with decompensated cirrhosis and ACLF who had been admitted with HRS-AKI and who had expired during that hospitalization.

\section{Methods}

This is a retrospective analysis, in which all the reports of postmortem kidney biopsy from January 2015 to January 2016, carried out at Institute of Liver and Biliary Sciences for patients with decompensated cirrhosis and ACLF who had been admitted with a diagnosis of HRS-AKI and who had expired during that hospitalization, were reviewed (Institutional Ethical Committee number: F.25/5/68/ILBS/AC/ 2014/302).

Patients eligible for this study included those with diagnosis of decompensated cirrhosis or ACLF and who had admitting diagnosis of HRS-AKI and who had expired during that hospitalization. Fig. 1 shows the selection of the patients for this study. During the study period, 451 patients died from liver diseases in the hospital, including 243 who had decompensated cirrhosis and 135 who had ACLF. The recruitment of patients was carried out by the hepatologist (MK) and the nephrologist (SLN), and kidney biopsy was performed by the nephrologist.

One-hundred-twenty-seven postmortem renal biopsies with adequate size ( $>1 \mathrm{~cm}$ in length) were available for analysis, and included 84 from patients with decompensated

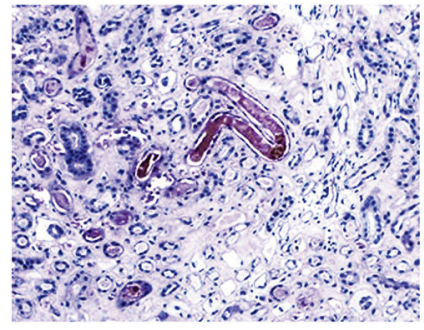

A

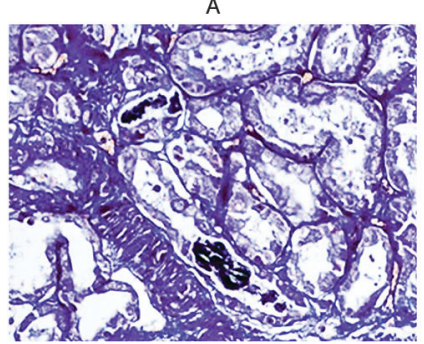

C

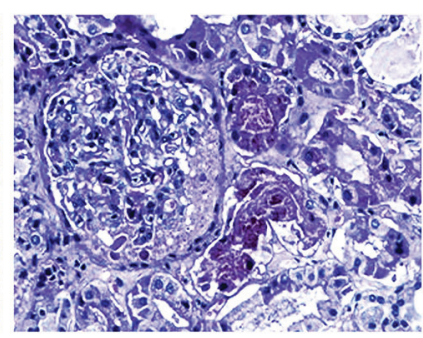

B

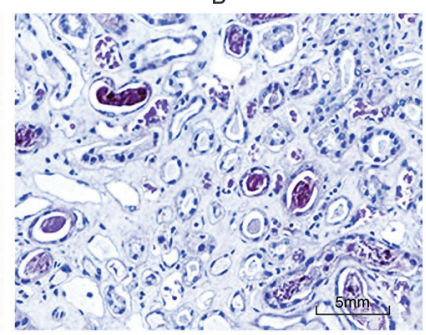

D
Fig. 1. Histopathologic findings in bile cast nephropathy. A) Bile casts in distal tubules with acute tubular necrosis (200X, hematoxylin and eosin staining). B) Bile casts $(3+)$ in proximal tubules (200X, hematoxylin and eosin staining). C) Greenish-colored bile cast (200X, Fouchet's stain). D) Bile casts are Pearl's stain-negative (no blue color) (200X, Pearl's stain). cirrhosis and 43 from patients with ACLF. The biopsies had been carried out within 30 minutes of declaration of death. Exclusion criteria for analysis were pregnant women, children and presence of any malignancies, including hepatocellular carcinoma, or chronic kidney disease.

The protocol of this study was approved by the Local Ethics Committee. Written informed consent was obtained from each patient's legal representative before the postmortem renal biopsy was taken.

\section{Definitions}

AKI was defined as an increase in serum creatinine of $\geq 50 \%$ from the stable baseline value in $<6$ months, which is known or presumed to have occurred within the prior 7 days, or an increase of $\geq 0.3 \mathrm{mg} / \mathrm{dL}$ in $<48 \mathrm{~h}$ or serum creatinine $>1.5 \mathrm{mg} / \mathrm{dL}$ at admission in the absence of known chronic kidney disease. ${ }^{15}$

To determine the cause of AKI, thorough history taking and careful physical examination were conducted to evaluate for potentially reversible and/or treatable causes for AKI, such as hypovolemia, hypotension, medications (i.e. diuretics, lactulose, and non-steroidal anti-inflammatory drugs), skin findings of cellulitis or line infection, and ongoing gastrointestinal losses (i.e. nausea, vomiting or diarrhea). Appropriate work-up for infections was determined by obtaining pancultures and performing chest radiography. Urinalysis and urine microscopy was performed to examine urine sediment and to detect findings suggestive of intrarenal injury such as tubular casts of ATN, microscopic hematuria, and red blood cells (RBCs) casts in glomerulonephritis or proteinuria.

\section{Diagnosis of HRS}

AKI diagnosis was made using the following criteria: 1) no improvement of serum creatinine after at least $48 \mathrm{~h}$ of diuretic withdrawal and volume expansion with albumin $(1 \mathrm{~g} / \mathrm{kg}$ body weight per day to maximum of $100 \mathrm{~g}$ albumin/day); 2) absence of shock; 3) no current or recent use of nephrotoxic drugs; and 4) no macroscopic signs of structural kidney disease as defined by absence of proteinuria $>500 \mathrm{mg} /$ day, microhematuria (>50 RBCs/high power field $(\mathrm{HPF}))$, and/or abnormal findings on renal ultrasound scan.

Patients with HRS-AKI were treated appropriately with antibiotics, vasopressors, albumin, renal replacement therapy and other supportive measures, as needed. Treatment with vasoconstrictors and albumin was started when serum creatinine went above $1.5 \mathrm{mg} / \mathrm{dL}$. Response to treatment was defined as no response (no regression of AKI), partial response (regression of AKI stage with a reduction of serum creatinine to $\geq 0.3 \mathrm{mg} / \mathrm{dL}$ above the baseline value) or full response (return of serum creatinine to a value within $0.3 \mathrm{mg} / \mathrm{dL}$ of the baseline value). ${ }^{15}$

ACLF was defined using the Asian Pacific Association for the Study of the Liver (APASL) criteria. The APASL defines ACLF as an acute hepatic insult manifesting as jaundice (serum bilirubin $\geq 5 \mathrm{mg} / \mathrm{dL}$ ) and coagulopathy (international normalization ratio of prothrombin time (INR) $\geq 1.5$ or prothrombin activity $<40 \%$ ) complicated within 4 weeks by clinical ascites and/or encephalopathy in a patient with previously diagnosed or undiagnosed chronic liver disease/ cirrhosis. ACLF is associated with a high 28 -day mortality. ${ }^{16}$ 
Nayak S.L. et al: Bile cast nephropathy in hepatorenal syndrome

\section{Renal biopsy examination}

Renal biopsy was performed using the 16-G Tru-cut biopsy needle under ultrasound guidance. Biopsy tissues were processed and subjected to light microscopy and immunofluorescence. We considered $>1 \mathrm{~cm}$ biopsy as adequate.

Bile casts were identified by light microscopy and confirmed by two stains (Fouchet's stain and Perl's stain). Bile casts were considered positive according to green color on Fouchet's staining and negative Perl's stain in at least one tubular lumen. Other types of bile pigmented casts (myoglobin, hemoglobin, hemosiderin and myeloma casts) were ruled out by appropriate clinical setting and tests. Immunohistochemical stains were used for myoglobin and hemoglobin; Prussian blue iron stain was used to exclude hemosiderin. Myeloma casts were ruled out by using the characteristic morphology of myeloma casts (fractured or sharp edged), serum and urine protein electrophoresis and absence of urinary Bence Jones protein.

The total number of bile cast tubules was counted in a minimum of 10 HPFs and the average number was per biopsy was calculated. Bile casts were graded as: $1+(1-5$ tubular bile casts in distal tubule); $2+$ ( $>5$ tubules with casts in distal tubules) and $3+$ (multiple casts both in proximal and distal tubules). We excluded the casts with red to dark red color, which were comprised of acellular material, sloughed epithelial cells and crystals. Acute tubular injury (ATI) was defined as tubular epithelium with attenuated cytoplasm or loss of proximal tubular brush borders or regenerative changes. Other details also recorded were as follows: interstitial inflammation, interstitial fibrosis, tubular atrophy, glomerular changes, arterionephrosclerosis, nephrocalcinosis, diabetic nephropathy, acute pyelonephritis, thrombotic microangiopathy, renal hemorrhage, membranoproliferative glomerulonephritis, ischemic glomerulopathy, collapsing glomerulopathy, obstructive nephropathy, tubulointerstitial nephritis, or Randall plaques.

Urine routine testing and microscopy examination was carried out and urinary sodium was also measured by spot testing. The glomerular filtration rate (GFR) was estimated using the equation for modification of diet in renal disease.

\section{Statistical analysis}

Statistical analysis was performed using the Statistical Package for Social Science (SPSS) for Windows, version 22 (IBM Corp, Armonk, NY, USA). Continuous variables were expressed as mean \pm SD or median (range) as appropriate, and categorical variables were expressed as percentage. Categorical variables were compared by chi-square test or Fisher's exact test as appropriate. Continuous variables were compared by Student's $t$-test or Mann-Whitney test as appropriate.

Multinomial logistic regression was used to find predictors of $\mathrm{BCN}$ on renal biopsy. Receiver operating characteristic (ROC) curve was drawn to find the best cut-off (at maximum of sensitivity plus specificity) of total and direct bilirubin to predict presence of $\mathrm{BCN}$ on renal biopsy.

All statistical tests were 2-tailed, and results were statistically significant when the $p$-value was less than 0.05 .
Results

\section{Baseline characteristics}

The baseline characteristics of the 127 patients are summarized in Table 1. The patients ranged in age from $10-81$ years old, with a median of 46 years old. There were 112 males $(88.2 \%)$ and 15 females (11.8\%). Among the total population, $84(66.1 \%)$ had decompensated cirrhosis and 43 $(33.9 \%)$ had ACLF. The most common likely precipitants of HRS-AKI were infection $(83 / 127,65.3 \%)$ and diuretic use $(41 / 127,32.3 \%)$.

\section{Characteristics of patients with decompensated cirrhosis and ACLF}

Table 1 shows the characteristics of patients with decompensated cirrhosis and ACLF. As expected, patients with ACLF had significantly higher levels of total bilirubin (median (range), $25.8(5.0-72.8) \mathrm{mg} / \mathrm{dL}$ vs. $6.2(1.0-44.3) \mathrm{mg} / \mathrm{dL}$; $p=0.01)$, INR (median (range), $2.48(1.5-7.08)$ vs. 1.9 $(1.1-9.0) ; p=0.01)$ and model for end-stage liver disease (MELD) (median (range), 37.0 (20.0-59.0) vs. 28.0 (12.0$56.0)$ vs. $24.2(8.2-56.27) ; p=0.01)$, as compared to the decompensated cirrhotics. BCN was found in $32 / 43(74.4 \%)$ of the patients with ACLF, as compared to 25/84 (29.7\%) of the patients with decompensated cirrhosis $(p<0.001)$. Urinary spot sodium levels and other urinary findings were comparable among the patients with ACLF and those with decompensated cirrhosis (Table 1 ). The causes of mortality were also comparable among the patients with ACLF and those with decompensated cirrhosis (Table 1).

\section{Characteristics of patients with and without BCN on postmortem renal biopsy}

BCN was detected in 57 of 127 (44.8\%) cases (Fig. 1). Table 2 shows the baseline characteristics of patients with and without BCN (bile casts) on renal biopsy. Fig. 2 shows the response rates to terlipressin and albumin treatment in patients with and without BCN on renal biopsy. Overall, 50 of $57(87.7 \%)$ of patients with BCN on kidney biopsy and 56 of $70(80.0 \%)$ of patients without BCN on kidney biopsy were nonresponsive to terlipressin and albumin treatment ( $p=0.244)$. Among the ACLF patients, 29 of $32(90.6 \%)$ of the patients with BCN on kidney biopsy and 6 of 11 (54.5\%) of the patients without $\mathrm{BCN}$ on kidney biopsy were nonresponsive to terlipressin and albumin treatment $(p=0.007)$. Among patients with decompensated cirrhosis, 21 of 25 $(84.0 \%)$ of the patients with BCN on kidney biopsy and 50 of $59(84.7 \%)$ of the patients without BCN on kidney biopsy were nonresponsive to terlipressin and albumin treatment $(p=0.931)$.

Patients with BCN had significantly higher levels of serum total bilirubin (median (range), $27.0(1.5-72.8) \mathrm{mg} / \mathrm{dL}$ vs. $8.1(1.0-32.7) \mathrm{mg} / \mathrm{dL} ; p<0.001)$, total leukocyte count (median (range), 25,000 (1200-60,800) per cu mm vs. $20,400(1000-54,900)$ per $\mathrm{cu} \mathrm{mm} ; p=0.01)$ and MELD score (median (range), 36.0 (18.0-59.0) vs. 28.0 (12.0$55.0) ; p=0.01$ ). Median (range) of creatinine, albumin and INR were not significantly different among patients with or without $\mathrm{BCN}$ on postmortem renal biopsy. Of the 57 patients with $\mathrm{BCN}, 32(56.1 \%)$ had ACLF, whereas only 11 of the 70 $(15.5 \%)$ patients without $\mathrm{BCN}$ had $\operatorname{ACLF}(p=0.001)$. There 
Nayak S.L. et al: Bile cast nephropathy in hepatorenal syndrome

Table 1. Baseline characteristics of patients with decompensated cirrhosis and acute on chronic liver failure

\begin{tabular}{|c|c|c|c|}
\hline Characteristic & $\begin{array}{l}\text { Decompensated cirrhosis } \\
(n=84)\end{array}$ & $\operatorname{ACLF}(n=43)$ & $p$ \\
\hline Age in years, mean $\pm S D$ & $49.0 \pm 11.4$ & $44.1 \pm 13.8$ & 0.04 \\
\hline Male sex, $n(\%)$ & $75(89.3 \%)$ & $37(86 \%)$ & 0.18 \\
\hline Total bilirubin in $\mathrm{mg} / \mathrm{dL}$, median (range) & $6.2(1.0-44.3)$ & $25.8(5.1-72.8)$ & 0.01 \\
\hline Direct bilirubin in $\mathrm{mg} / \mathrm{dL}$, median (range) & $3.0(0.2-27.6)$ & $9.0(4.3-42.4)$ & 0.01 \\
\hline AST in IU/L, median (range) & $90.0(20.0-1170.0)$ & $140.0(36.0-6210.0)$ & 0.12 \\
\hline ALT in IU/L, median (range) & $42.5(9.0-913.0)$ & $59.0(17.0-785.0)$ & 0.05 \\
\hline ALP in IU/L, median (range) & $120.5(33.0-911.0)$ & $108.0(31.0-428.0)$ & 0.19 \\
\hline GGT in IU/L, median (range) & $46.5(12.0-1186.0)$ & $74.0(19.0-601.0)$ & 0.17 \\
\hline Total protein in $\mathrm{g} / \mathrm{L}$, median (range) & $6.6(4.4-9.5)$ & $6.5(3.8-8.3)$ & 0.21 \\
\hline Albumin in $\mathrm{g} / \mathrm{L}$, median (range) & $2.2(1.1-3.8)$ & $2.3(1.0-3.3)$ & 0.94 \\
\hline $\mathrm{Hb}$ in $\mathrm{g} / \mathrm{dL}$, mean $\pm \mathrm{SD}$ & $8.4 \pm 1.8$ & $8.4 \pm 1.6$ & 0.92 \\
\hline Platelet count in $10^{9} / \mathrm{L}$, median (range) & $45.0(9.0-553.0)$ & $59.0(13.0-261.0)$ & 0.43 \\
\hline TLC in per cu mm, median (range) & $20.8(1.0-54.9)$ & $23.9(1.8-60.8)$ & 0.04 \\
\hline $\begin{array}{l}\text { Serum creatinine in } \mathrm{mg} / \mathrm{dL} \text {, } \\
\text { median (range) }\end{array}$ & $1.7(1.5-8.4)$ & $2.5(1.5-10.3)$ & 0.92 \\
\hline MELD, median (range) & $28.0(12.0-56.0)$ & $37.0(20.0-59.0)$ & 0.01 \\
\hline INR, median (range) & $1.9(1.1-9.0)$ & $2.48(1.5-7.0)$ & 0.01 \\
\hline $\mathrm{BCN}$ as bile casts present, $n(\%)$ & $25(29.7 \%)$ & $32(74.4 \%)$ & $<0.001$ \\
\hline $\begin{array}{l}\text { Etiology of decompensated cirrhosis, } \\
n(\%) \\
\text { ALD } \\
\text { CHBV infection } \\
\text { CHCV infection } \\
\text { NASH cirrhosis }\end{array}$ & $\begin{array}{l}70(83.3 \%) \\
10(11.9 \%) \\
2(2.4 \%) \\
2(2.4 \%)\end{array}$ & Not applicable & \\
\hline $\begin{array}{l}\text { Etiology of ACLF; Chronic/Acute, } n(\%) \\
\text { ALD/Alcoholic hepatitis } \\
\text { CHBV infection/Reactivation of HBV } \\
\text { NASH cirrhosis/HEV } \\
\text { NASH cirrhosis/DILI }\end{array}$ & Not applicable & $\begin{array}{l}25(58.1 \%) \\
12(27.9 \%) \\
4(9.3 \%) \\
2(4.7 \%)\end{array}$ & \\
\hline $\begin{array}{l}\text { Likely precipitant of HRS, } n(\%) \\
\text { Infection } \\
\text { Diuretic use } \\
\text { Others }\end{array}$ & $\begin{array}{l}52(61.9 \%) \\
30(35.7 \%) \\
2(2.4 \%)\end{array}$ & $\begin{array}{l}31(72.1 \%) \\
11(25.6 \%) \\
1(2.3 \%)\end{array}$ & \\
\hline $\begin{array}{l}\text { Cause of death, } n(\%) \\
\text { Hepatic failure } \\
\text { Sepsis with multiorgan failure } \\
\text { Cardiac event } \\
\text { GI hemorrhage } \\
\text { Hepatic failure + Encephalopathy } \\
\text { Hepatic failure + GI hemorrhage }\end{array}$ & $\begin{array}{l}36(42.9 \%) \\
18(21.4 \%) \\
2(2.4 \%) \\
4(4.8 \%) \\
16(19.0 \%) \\
8(9.5 \%)\end{array}$ & $\begin{array}{l}17(39.5 \%) \\
11(25.6 \%) \\
2(4.7 \%) \\
0(0 \%) \\
7(16.3 \%) \\
6(14 \%)\end{array}$ & 0.634 \\
\hline \multicolumn{4}{|l|}{ Urinary findings } \\
\hline Spot sodium in $\mathrm{mEq} / \mathrm{L}$, median (range) & $7.0(3.0-10.0)$ & $8.0(3.0-10)$ & 0.275 \\
\hline RTE cells/HPF as $0 / 1-5, n(\%)$ & $56(66.7 \%) / 28(33.3 \%)$ & $32(74.4 \%) / 11(25.6 \%)$ & 0.421 \\
\hline WBC/HPF as $0 / 1-5 / 6-20, n(\%)$ & $65(77.4 \%) / 12(14.3 \%) / 7(8.3 \%)$ & $33(76.7 \%) / 5(11.6 \%) / 5(11.6 \%)$ & 0.787 \\
\hline RTE cell casts/HPF as $0 / 1-5, n(\%)$ & $78(92.9 \%) / 6(7.1 \%)$ & $39(90.7 \%) / 4(9.3 \%)$ & 0.733 \\
\hline Hyaline casts/HPF as $0 / 1-5 / 6-20, n(\%)$ & $75(89.3 \%) / 4(4.8 \%) / 5(6.0 \%)$ & $37(86.0 \%) / 4(9.3 \%) / 2(4.7 \%)$ & 0.591 \\
\hline $\mathrm{RBC} / \mathrm{HPF}$ as $0 / 1-5 / 6-20, n(\%)$ & $72(85.7 \%) / 12(14.3 \%)$ & $37(86.0 \%) / 6(14.0 \%)$ & 1.0 \\
\hline Protein in Nil/1+, $n(\%)$ & $74(88.1 \%) / 10(11.9 \%)$ & $37(86.0 \%) / 6(14.0 \%)$ & 0.781 \\
\hline
\end{tabular}

Abbreviations: INR, international normalization ratio of prothrombin time; AST, aspartate transaminase; ALT, alanine transaminase; ALP, alkaline phosphatase; Hb, hemoglobin; TLC, total leukocyte count; MELD, model for end-stage liver disease; ACLF, acute on chronic liver failure; ALD, alcoholic liver disease; CHBV, chronic hepatitis B virus; $\mathrm{CHCV}$, chronic hepatitis C virus; NASH, nonalcoholic steatohepatitis; HEV, hepatitis E virus; DILI, drug-induced liver injury; BCN, bile cast nephropathy; GI, gastrointestinal; RTE, renal tubular epithelial; WBC, white blood cells; RBC, red blood cells; HPF, high power field. 
Nayak S.L. et al: Bile cast nephropathy in hepatorenal syndrome

Table 2. Baseline characteristics of patients with and without BCN (bile casts) on postmortem renal biopsy

\begin{tabular}{|c|c|c|c|}
\hline Characteristic & $\begin{array}{l}\text { Bile cast nephropathy } \\
\text { (Bile casts present) } \\
n=57\end{array}$ & $\begin{array}{l}\text { No bile cast nephropathy } \\
\text { (Bile casts absent) } \\
n=70\end{array}$ & $p$ \\
\hline Age in years, median (range) & $40(10-74)$ & $52(27-81)$ & 0.01 \\
\hline Male sex, $n(\%)$ & $52(91.2 \%)$ & $60(85.7 \%)$ & 0.54 \\
\hline Total bilirubin in mg/dL, median (range) & $27.0(1.5-72.8)$ & $8.1(1.0-32.7)$ & $<0.001$ \\
\hline Direct bilirubin in $\mathrm{mg} / \mathrm{dL}$, median (range) & $16.3(0.2-45.8)$ & $2.6(0.2-14.8)$ & $<0.001$ \\
\hline AST in IU/L, median (range) & $118.0(36.0-6210.0)$ & $83.0(20.0-1170.0)$ & 0.18 \\
\hline ALT in IU/L, median (range) & $48.0(17.0-785.0)$ & $44.0(9.0-913.0)$ & 0.39 \\
\hline ALP in IU/L, median (range) & $127.0(31.0-290.0)$ & $109.0(33.0-911.0)$ & 0.42 \\
\hline GGT in IU/L, median (range) & $74.0(12.0-601.0)$ & $41.0(14.0-1186.0)$ & 0.35 \\
\hline Total protein in $\mathrm{g} / \mathrm{L}$, median (range) & $6.5(3.8-8.3)$ & $6.6(4.4-9.5)$ & 0.24 \\
\hline Albumin in $\mathrm{g} / \mathrm{L}$, median (range) & $2.1(1.0-3.4)$ & $2.3(1.1-3.8)$ & 0.09 \\
\hline $\mathrm{Hb}$ in $\mathrm{g} / \mathrm{dL}$, median (range) & $7.9(5.5-12.8)$ & $8.3(3.4-12.5)$ & 0.43 \\
\hline Platelet count in $10^{9} / \mathrm{L}$, median (range) & $56(10-553)$ & $50.5(9-388)$ & 0.33 \\
\hline TLC in thousand per cu mm, median (range) & $25(1.2-60.8)$ & $20.4(1.0-54.9)$ & 0.01 \\
\hline Serum creatinine in $\mathrm{mg} / \mathrm{dL}$, median (range) & $2.6(1.5-10.3)$ & $1.7(1.5-8.4)$ & 0.83 \\
\hline INR, median (range) & $2.3(1.1-6.5)$ & $1.9(1.1-9.0)$ & 0.12 \\
\hline MELD score, median (range) & $36.0(18.0-59.0)$ & $28.0(12.0-55.0)$ & 0.01 \\
\hline ACLF/Decompensated cirrhosis, $n(\%)$ & $32(56.1 \%) / 25(43.9 \%)$ & $11(15.7 \%) / 59(84.3 \%)$ & $<0.001$ \\
\hline $\begin{array}{l}\text { Etiology of decompensated cirrhosis }(n=84) \text {, } \\
n(\%) \\
\text { ALD } \\
\text { CHBV infection } \\
\text { CHCV infection } \\
\text { NASH cirrhosis }\end{array}$ & $\begin{array}{l}\text { Overall: } 23 / 84(27.4 \%) \\
\begin{array}{l}20 / 70(28.6 \%) \\
3 / 10(30.0 \%) \\
1 / 2(50.0 \%) \\
1 / 2(50.0 \%)\end{array}\end{array}$ & $\begin{array}{l}61 / 84(72.6 \%) \\
50 / 70(71.4 \%) \\
7 / 10(70.0 \%) \\
1 / 2(50.0 \%) \\
1 / 2(50.0 \%)\end{array}$ & 0.831 \\
\hline $\begin{array}{l}\text { Etiology of ACLF; Chronic/Acute, }(n=43) \text {, } \\
n(\%) \\
\text { ALD/Alcoholic hepatitis } \\
\text { CHBV infection/Reactivation of HBV } \\
\text { NASH cirrhosis/HEV } \\
\text { NASH cirrhosis/DILI }\end{array}$ & $\begin{array}{l}\text { Overall: } 31 / 43(72.1 \%) \\
\text { 19/25(76.0\%) } \\
9 / 12(75.0 \%) \\
3 / 4(75.0 \%) \\
1 / 2(50.0 \%)\end{array}$ & $\begin{array}{l}12 / 43(27.9 \%) \\
6 / 25(24.0 \%) \\
3 / 12(25.0 \%) \\
1 / 4(25.0 \%) \\
1 / 2(50.0 \%)\end{array}$ & 0.662 \\
\hline $\begin{array}{l}\text { Cause of death, } n(\%) \\
\text { Hepatic failure } \\
\text { Sepsis with multiorgan failure } \\
\text { Cardiac event } \\
\text { GI hemorrhage } \\
\text { Hepatic failure + Encephalopathy } \\
\text { Hepatic failure + GI hemorrhage }\end{array}$ & $\begin{array}{l}24(42.1 \%) \\
18(31.6 \%) \\
2(3.5 \%) \\
1(1.8 \%) \\
7(12.3 \%) \\
5(8.8 \%)\end{array}$ & $\begin{array}{l}29(41.4 \%) \\
11(15.7 \%) \\
2(2.9 \%) \\
3(4.3 \%) \\
16(22.9 \%) \\
9(12.9 \%)\end{array}$ & 0.255 \\
\hline \multicolumn{4}{|l|}{ Urinary findings } \\
\hline $\begin{array}{l}\text { Spot sodium in } \mathrm{mEq} / \mathrm{L}, \text { median (range) } \\
\text { RTE cells/HPF as } 0 / 1-5, n(\%) \\
\text { WBC/HPF as } 0 / 1-5 / 6-20, n(\%)\end{array}$ & $\begin{array}{l}8.0(4.0-10.0) \\
39(68.4 \%) \\
43(75.4 \%) / 7(12.3 \%) / 7 \\
(12.3 \%)\end{array}$ & $\begin{array}{l}7.5(3.0-10.0) \\
49(70 \%) \\
55(78.6 \%) / 10(14.3 \%) / 5 \\
(7.1 \%)\end{array}$ & $\begin{array}{l}0.935 \\
0.849 \\
0.603\end{array}$ \\
\hline RTE cell casts/HPF as $0 / 1-5, n(\%)$ & $52(91.2 \%) / 5(8.8 \%)$ & $65(92.9 \%) / 5(7.1 \%)$ & 0.752 \\
\hline Hyaline casts/HPF as $0 / 1-5 / 6-20, n(\%)$ & $\begin{array}{l}49(86.0 \%) / 5(8.8 \%) / 3 \\
(5.2 \%)\end{array}$ & $\begin{array}{l}63(90.0 \%) / 3(4.3 \%) / 4 \\
(5.7 \%)\end{array}$ & 0.585 \\
\hline $\mathrm{RBC} / \mathrm{HPF}$ as $0 / 1-5 / 6-20, n(\%)$ & $48(84.2 \%) / 9(15.8 \%)$ & $61(87.1 \%) / 9(12.9 \%)$ & 0.799 \\
\hline Protein as $\mathrm{Nil} / 1+, n(\%)$ & $48(84.2 \%) / 9(15.8 \%)$ & $63(90.0 \%) / 7(70.0 \%)$ & 0.781 \\
\hline
\end{tabular}

Abbreviations: INR, international normalization ratio of prothrombin time; AST, aspartate transaminase; ALT, alanine transaminase; ALP, alkaline phosphatase; $\mathrm{Hb}$, hemoglobin; TLC, total leukocyte count; MELD, model for end-stage liver disease; ACLF, acute on chronic liver failure; ALD, alcoholic liver disease; CHBV, chronic hepatitis B virus; $\mathrm{CHCV}$, chronic hepatitis C virus; NASH, nonalcoholic steatohepatitis; HEV, hepatitis E virus; DILI, drug-induced liver injury; BCN, bile cast nephropathy; GI, gastrointestinal; RTE, renal tubular epithelial; WBC, white blood cells; RBC, red blood cells; HPF, high power field. 
Nayak S.L. et al: Bile cast nephropathy in hepatorenal syndrome

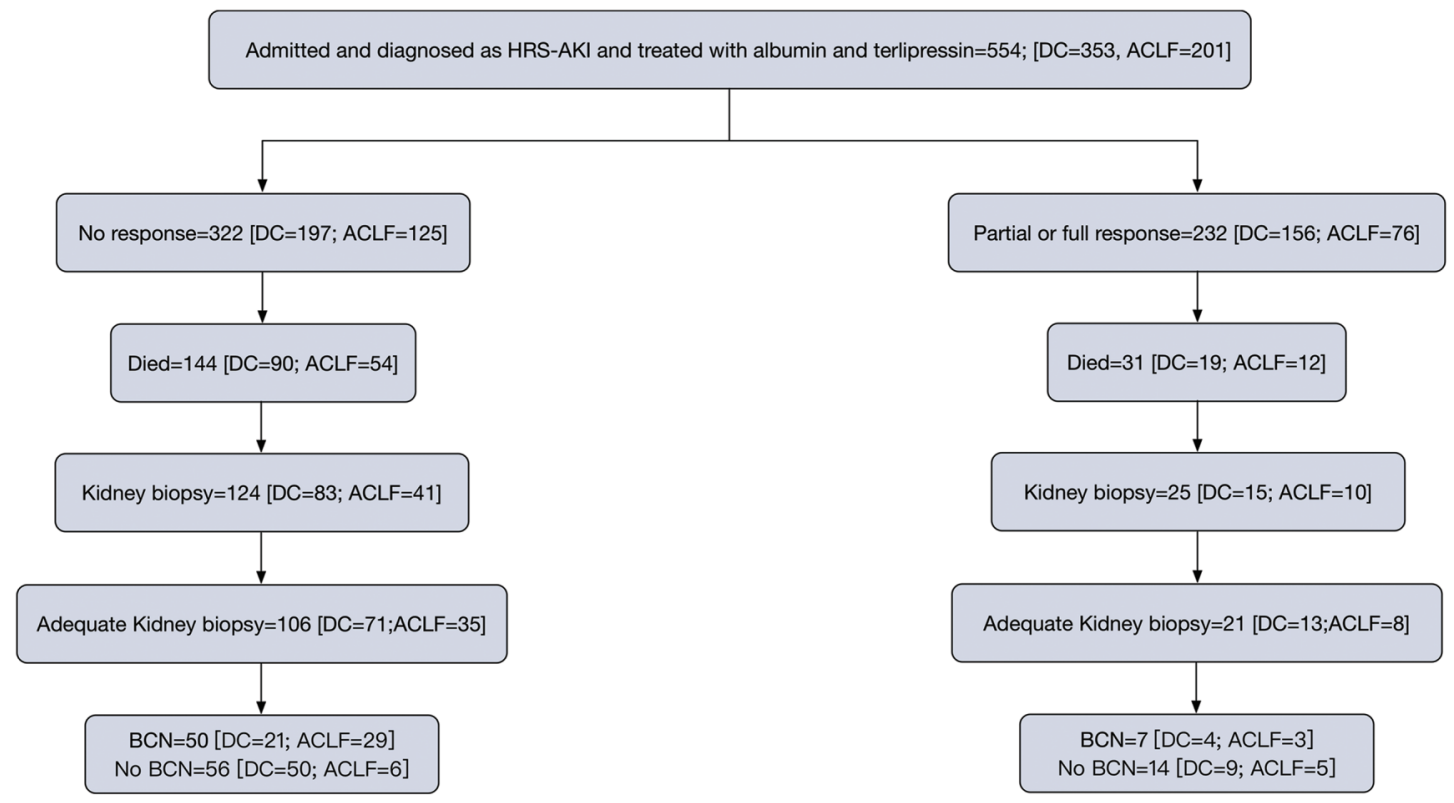

Note:During the year 451 patients died in the hospital due to liver diseases [243 had decompensated cirrhosis and 135 had acute on chronic liver failure]

Fig. 2. Selection of patients for the study.

Abbreviations: DC, decompensated cirrhosis; ACLF, acute on chronic liver failure; BCN, bile cast nephropathy.

was no difference in presence or absence of BCN according to etiology of liver disease.

Urinary spot sodium levels and other urinary findings were comparable among patients with or without BCN on postmortem renal biopsy (Table 2 ). The causes of mortality were also comparable among patients with or without $\mathrm{BCN}$ on postmortem renal biopsy (Table 2).

\section{Predictors of BCN}

Table 3 shows the predictors of $\mathrm{BCN}$ on postmortem renal biopsy. On univariate analysis, total bilirubin (OR (95\% CI): $1.12(1.078-1.170) ; p<0.001)$, direct bilirubin (OR (95\% CI): $1.208(1.132-1.289) ; p<0.001)$, total leukocyte count (OR (95\% CI): $1.048(1.017-1.079) ; p=0.002)$, MELD (OR (95\% CI): $1.089(1.046-1.134) ; p<0.001)$ and presence of ACLF (OR (95\% CI): 6.8 (2.995-15.736); $p<0.001$ ) were found to be significant predictors of $\mathrm{BCN}$ on postmortem renal biopsy.

On multivariate analysis, direct bilirubin (OR (95\% CI): $1.27(1121-1.698) ; p<0.001)$ and presence of ACLF (OR (95\% CI): $2.603(1.054-7.111) ; p=0.041)$ were found to be significant predictors of $\mathrm{BCN}$ on postmortem renal biopsy. ROC curve was drawn to find the best cut-off of total bilirubin and direct bilirubin to predict presence of BCN on renal biopsy. The best cut-off for total bilirubin for predicting $\mathrm{BCN}$ on renal biopsy was $14.7 \mathrm{mg} / \mathrm{dL}$ (area under the ROC $(A U R O C)=0.830 ; 95 \%$ CI: 0.759-0.901; $p<0.001$ ) with sensitivity of $70.2 \%$ and specificity of $78.6 \%$. The best cutoff for direct bilirubin for predicting $\mathrm{BCN}$ on renal biopsy was $6.1 \mathrm{mg} / \mathrm{dL}$ (AUROC = 0.839; 95\% CI: 0.768-0.910; $p<0.001$ ) with sensitivity of $75.4 \%$ and specificity of $74.3 \%$.

\section{Other histological findings in patients with BCN}

Bile casts were present in 57 of the 127 (44.8\%) autopsy kidney specimens. Grade $1+(1-5$ tubular bile casts in distal tubule), $2+$ ( $>5$ tubules with casts in distal tubules) and 3+ (multiple casts both in proximal and distal tubules) bile casts were seen in $22 / 57$ (38.6\%), 15/57 (26.3\%) and 20/57 $(35.1 \%)$ of the patients. Interstitial edema, interstitial fibrosis, and tubular atrophy were seen in $11 / 57$ (19.3\%), 6/57 $(10.5 \%)$ and $1 / 57(1.7 \%)$ patients respectively. Vascular changes were not seen in any of the biopsy specimens. The severity of bile cast formation did not reveal an apparent relationship with other laboratory or clinical data.

Table 3. Univariate and multivariate predictors of BCN

\begin{tabular}{lll}
\hline Variable & $\begin{array}{l}\text { Univariate } \\
\text { OR }(95 \% \mathrm{CI}), p\end{array}$ & $\begin{array}{l}\text { Multivariate } \\
\text { OR }(95 \% \mathrm{CI}), p\end{array}$ \\
\hline Total & $1.120(1.078-1.170)$, & $0.956(0.801-1.141)$, \\
bilirubin & $<0.001$ & 0.956 \\
Direct & $1.208(1.132-1.289)$, & $1.27(1.121-1.698)$, \\
bilirubin & $<0.001$ & $<0.001$ \\
TLC & $1.048(1.017-1.079)$, & $1.020(0.983-1.059)$, \\
& $<0.002$ & 0.287 \\
MELD & $1.089(1.046-1.134)$, & $0.999(0.946-1.054)$, \\
& $<0.001$ & 0.685 \\
ACLF & $6.800(2.995-15.736)$, & $2.603(1.054-7.111)$, \\
& $<0.001$ & 0.041 \\
\hline
\end{tabular}

Abbreviations: BCN, bile cast nephropathy; TLC, total leukocyte count; MELD, model for end-stage liver disease; ACLF, acute on chronic liver failure. 
Nayak S.L. et al: Bile cast nephropathy in hepatorenal syndrome

\section{Discussion}

HRS-AKI occurs frequently in patients with ESLD and ACLF and portends a poor prognosis. The current paradigm of renal dysfunction in HRS-AKI is primarily attributed to marked splanchnic/systemic vasodilatation that results in a reduction of the GFR. Only about $40-50 \%$ of patients with HRS-AKI have favorable response to albumin and terlipressin treatment.

$\mathrm{BCN}$, although known for many decades, has remained a relatively overlooked entity. Its exact pathophysiological mechanism is as yet unknown. Serum bile and bilirubin levels increase in the setting of liver failure. It is believed that hyperbilirubinemia causes damage to the kidney in several ways. First, bile casts cause tubular obstruction and, second, the presence of high concentrations of bile in the renal tubules may be toxic to tubular cells, resulting in impairment of tubular function. ${ }^{17}$ It is still unclear whether renal injury per se leads to deposition of bile casts or it's the primary deposition of bile casts that leads to renal injury.

Bilirubin itself is a potent vasodilator that can lead to obligatory loss of water, up to $1.5 \mathrm{~L}$, along with salt loss. ${ }^{18}$ In the animal model, it has been shown that conjugated bilirubin is toxic to cells, causing uncoupling of oxidative phosphorylation and giving rise to less production of adenosine triphosphate per glucose, thereby leading to cellular hypoxia. Moreover, the renal parenchyma becomes sensitized to ischemic damage. ${ }^{19}$ After glomerular filtration, free conjugated bilirubin can enter the tubular cells from the lumen, causing direct damage.

Besides bilirubin, other cholephils have also been postulated as important in pathogenesis of BCN. A landmark study showed that renal tubular epithelial injury, intratubular cast formation and tubulointerstitial fibrosis can occur in common bile duct-ligated mice, which is not only related to the duration of ligation but also to the toxic effect of bile acids. ${ }^{20}$ Studies have shown that infusion of bile acids to animals precipitates renal failure, with formation of bile casts in kidney, which could be detected in urine. ${ }^{21,22}$ Low water solubility of bile acids within an acidic microenvironment of the distal nephron facilitates cast formation that causes tubular obstruction and subsequent AKI. ${ }^{6}$

In this study, we found $\mathrm{BCN}$ in postmortem renal biopsies for 57 of 127 (44.8\%) patients, including 25/84 (29.7\%) of patients with decompensated cirrhosis and $32 / 43(74.4 \%)$ of patients with ACLF. The patients studied had been admitted with HRS-AKI that did not respond to treatment and they had died during the hospitalization. There are limited clinical studies of BCN in patients with decompensated cirrhosis and ACLF. Van Slambrouck et al. ${ }^{6}$ reported a study of 41 autopsy cases and 3 patients with kidney biopsies, in which they evaluated cases of cholemic nephropathy in jaundiced patients with cirrhosis $(n=23)$, cholestatic/obstructive jaundice $(n=13)$, severe acute liver dysfunction $(n=5)$ and hemolytic jaundice $(n=2)$. Thirteen of the total 44 patients in the study were clinically classified as having HRS; however, 11 $(85 \%)$ out of these 13 patients showed bile casts on kidney histology. ${ }^{6}$ Another case report described a patient with known alcoholic cirrhosis presenting with severe jaundice (total bilirubin of $23.1 \mathrm{mg} / \mathrm{dL}$ ), as well as AKI and anuria. Initial urinalysis had suggested intrinsic renal disease but percutaneous kidney biopsy (after coagulopathy correction) performed a few days after initiation of dialysis demonstrated presence of bile casts involving proximal tubules along with acute tubular injury. ${ }^{12}$

In this study, BCN was found in $32 / 43(74.4 \%)$ of patients with ACLF, as compared to $25 / 84(29.7 \%)$ of patients with decompensated cirrhosis $(p<0.001)$. Presence of ACLF was also found to be an independent predictor of $\mathrm{BCN}$ on renal biopsy (OR (95\% CI): 2.603 (1.054-7.111); $p=0.041$ ). There are no studies of BCN in patients with ACLF and HRSAKI. It is not known when bile cast formation occurs during the development of HRS-AKI, but the likelihood of bile cast formation increases with prolonged exposure to high levels of bilirubin. ${ }^{6}$ It is believed that patients with bilirubin concentrations $>20 \mathrm{mg} / \mathrm{dL}$ are more prone to developing bile cast nephropathy. 17

In the current study, patients with BCN had significantly higher levels of serum total bilirubin (median (range), 27.0 (1.5-72.8) $\mathrm{mg} / \mathrm{dL}$ vs. $8.1(1.0-32.7) \mathrm{mg} / \mathrm{dL} ; p<0.001)$ and MELD score (median (range), 32.9 (10.9-57.6) vs. 24.06 (8.2-56.2; $p=0.01)$, as compared to patients who did not have BCN. On multivariate analysis, direct bilirubin (OR (95\% CI): $1.27(1121-1.698) ; p<0.001)$ and presence of ACLF (OR (95\% CI): 2.603 (1.054-7.111); $p=0.041$ ) were found to be significant predictors of $\mathrm{BCN}$ on postmortem renal biopsy.

The best cut-off for total bilirubin for predicting $\mathrm{BCN}$ on renal biopsy was $14.7 \mathrm{mg} / \mathrm{dL}$ (AUROC $=0.830 ; 95 \% \mathrm{CI}$ : $0.759-0.901 ; p<0.001$ ) with sensitivity of $70.2 \%$ and specificity of $78.6 \%$. The best cut-off for direct bilirubin for predicting BCN on renal biopsy was $6.1 \mathrm{mg} / \mathrm{dL}$ (AUROC $=0.839$; 95\% CI: $0.768-0.910 ; p<0.001$ ) with sensitivity of $75.4 \%$ and specificity of $74.3 \%$. In the study by Van Slambrouck et ${ }^{2} .{ }^{6}{ }^{6}$ the mean total serum bilirubin and direct bilirubin was $26.2 \mathrm{mg} / \mathrm{dL}$ and $16.3 \mathrm{mg} / \mathrm{dL}$ among patients with bile casts compared to $15.1 \mathrm{mg} / \mathrm{dL}$ and $9.2 \mathrm{mg} / \mathrm{dL}$ among patients without $\operatorname{BCN}(p=0.001$ and 0.003 respectively). Also in that study, HRS patients (which constituted $30 \%$ of the total 44 patients) with BCN had higher levels of total serum bilirubin (though not to a statistically significant extent) compared to patients without $\mathrm{BCN}$. We were not able to measure serum bile acids and it would be interesting to assess the role of serum bile acids as a predictor of BCN in future studies on HRS patients.

This study also did not find any relation of etiology of liver disease with the presence or absence of BCN. The study by Van Slambrouck et al., ${ }^{6}$ found that all 10 patients in their study with cirrhosis due to alcohol abuse had bile casts, whereas none of the 5 patients with cirrhosis secondary to hepatitis $C$ virus had bile casts.

The role $\mathrm{BCN}$ plays in pathogenesis, progression and response to vasoconstrictor therapy of HRS-AKI remains elusive. There has been limited clinical effectiveness of terlipressin and albumin treatment in HRS-AKI, with a reported response rate of about $40-50 \% .{ }^{23-25}$ The renal dysfunction in HRS is mainly attributed to marked splanchnic vasodilatation and renal vasoconstriction leading to drop in GFR. However, the impact of renal bile casts in such a scenario has not been investigated. Bile casts could be one of the mechanisms leading to no response or delayed response to standard care of treatment in HRS-AKI.

The likelihood of bile cast formation increases with prolonged exposure to high levels of bilirubin. This also points towards the fact that HRS-AKI probably represents a mixed bag of conditions, and BCN could be at least one part of it. Alternatively, it has also been suggested that bilirubin 
casts, noted in patients succumbing to liver failure and hepatorenal syndrome, might reflect reduced GFR and diminished washout of casts, rather than play a role in the pathogenesis of this disorder. ${ }^{26}$

The presence of this entity can also be a deciding factor in allocation of kidneys in cirrhotics. Patients with bile pigment nephropathy may or may not-if the bile casts have been present for a long time-regain normal kidney function after LT following the improvement of liver function.

$\mathrm{BCN}$ may be an overlooked condition that might at least contribute to AKI and/or renal dysfunction and their response to drugs in patients with severe liver disease, as kidney biopsy or autopsy studies are needed for the diagnosis of $B C N$. In the absence of renal biopsy, the diagnosis of HRS remains difficult and is essentially a diagnosis of exclusion. Due to concurrent coagulopathy and thrombocytopenia in advanced cirrhosis or ACLF, renal biopsy carries a high risk of internal bleeding and is rarely performed.

In one study of 55 patients with cirrhosis undergoing transjugular renal biopsy, 8 patients developed internal bleeding and 4 had perinephric hematoma. ${ }^{27}$ However, one recent study showed transjugular kidney biopsy to be relatively safe. ${ }^{28}$ Although biopsy is the gold standard test to recognize BCN, a few studies have found bile cast in urine, including one study on severe malaria with jaundice and renal failure. ${ }^{17}$ Our study did not find bile casts in urine in any of the patients, as this was not specifically looked for in urine and the proper procedure for assessing bile casts in urine was not followed (i.e. centrifugation of $10 \mathrm{~mL}$ of urine at $2000 \mathrm{rpm}$ for 20 minutes).

There has been recent interest in AKI urinary biomarkers like neutrophil gelatinase-associated lipocalin, kidney injury molecule- 1 , and $\pi$ - and $\alpha$-glutathione-S-transferases in evaluating patients with AKI in liver diseases. ${ }^{29}$ In future studies, it will be interesting to determine these urinary biomarkers for correlation with histological findings in patients with HRS-AKI.

There are a few limitations of this study. This was a retrospective observational study and it's difficult to prove the causal association between BCN and nonresponsiveness to therapy in HRS-AKI patients. Although, in this study among ACLF patients, 29 of $32(90.6 \%)$ of patients with BCN on kidney biopsy and 6 of $11(54.5 \%)$ of patients without $\mathrm{BCN}$ on kidney biopsy were nonresponders to terlipressin and albumin treatment $(p=0.007)$, whereas among patients with decompensated cirrhosis, 21 of $25(84.0 \%)$ of patients with BCN on kidney biopsy and 50 of $59(84.7 \%)$ of patients without $\mathrm{BCN}$ on kidney biopsy were nonresponders to terlipressin and albumin treatment $(p=0.931)$. The overrepresentation of nonresponders to vasoconstrictor therapy among ACLF patients (but not among decompensated cirrhosis patients) with $\mathrm{BCN}$ as compared to those without $\mathrm{BCN}$ on kidney biopsy may be due to the fact that a rapid rise in bilirubin levels occurs during ACLF, as compared to decompensated cirrhosis. However, this was not the appropriate study design to address the issue of whether patients with $\mathrm{BCN}$ have lower response rate to vasoconstrictor therapy, as compared to patients without $\mathrm{BCN}$, because it is not known what proportion of patients who responded to therapy had $\mathrm{BCN}$, and the study design of present study had selection bias towards nonresponders, who were more likely to die.

\section{Conclusions}

In conclusion, $\mathrm{BCN}$ was found in $74.4 \%$ of patients with ACLF and $29.7 \%$ of patients with decompensated cirrhosis who had been hospitalized with an admitting diagnosis of HRS-AKI and who had expired during that hospitalization and underwent postmortem renal biopsy. On multivariate analysis, total serum bilirubin and presence of ACLF were found to be significant predictors of $\mathrm{BCN}$ on postmortem renal biopsy.

\section{Conflict of interest}

The authors have no conflict of interests related to this publication.

\section{Author contributions}

Conceived and designed the study, acquired data, carried out the statistical analysis and drafted the initial manuscript (SLN, MK), interpreted the pathology slides (CB, AR).

\section{References}

[1] du Cheyron D, Bouchet B, Parienti JJ, Ramakers M, Charbonneau P. The attributable mortality of acute renal failure in critically ill patients with liver cirrhosis. Intensive Care Med 2005;31:1693-1699. doi: 10.1007/s00134005-2842-7.

[2] Moreau R, Durand F, Poynard T, Duhamel C, Cervoni JP, Ichaï P, et al. Terlipressin in patients with cirrhosis and type 1 hepatorenal syndrome: a retrospective multicenter study. Gastroenterology 2002;122:923-930. doi: 10.1053/gast.2002.32364

[3] Garcia-Tsao G, Parikh CR, Viola A. Acute kidney injury in cirrhosis. Hepatology 2008;48:2064-2077. doi: 10.1002/hep.22605.

[4] Ginès P, Schrier RW. Renal failure in cirrhosis. N Engl J Med 2009;361: 1279-1290. doi: 10.1056/NEJMra0809139.

[5] Betjes MG, Bajema I. The pathology of jaundice-related renal insufficiency: cholemic nephrosis revisited. J Nephrol 2006;19:229-233.

[6] van Slambrouck CM, Salem F, Meehan SM, Chang A. Bile cast nephropathy is a common pathologic finding for kidney injury associated with severe liver dysfunction. Kidney Int 2013;84:192-197. doi: 10.1038/ki.2013.78.

[7] Krones E, Wagner M, Eller K, Rosenkranz AR, Trauner M, Fickert P. Bile acid-induced cholemic nephropathy. Dig Dis 2015;33:367-375. doi: 10. $1159 / 000371689$.

[8] Uslu A, Taşli FA, Nart A, Postaci H, Aykas A, Bati H, et al. Human kidney histopathology in acute obstructive jaundice: a prospective study. Eur J Gastroenterol Hepatol 2010;22:1458-1465. doi: 10.1097/MEG. Ob013e32833f71f6.

[9] Luciano RL, Castano E, Moeckel G, Perazella MA. Bile acid nephropathy in a bodybuilder abusing an anabolic androgenic steroid. Am J Kidney Dis 2014; 64:473-476. doi: 10.1053/j.ajkd.2014.05.010.

[10] Song J, Chang A. Jaundice-associated acute kidney injury. NDT Plus 2009; 2:82-83. doi: 10.1093/ndtplus/sfn149.

[11] Bredewold OW, de Fijter JW, Rabelink T. A case of mononucleosis infectiosa presenting with cholemic nephrosis. NDT Plus 2011;4:170-172. doi: 10. 1093/ndtplus/sfr038.

[12] Sequeira A, Gu X. Bile cast nephropathy: an often forgotten diagnosis. Hemodial Int 2015;19:132-135. doi: 10.1111/hdi.12169.

[13] Salerno F, Gerbes A, Ginès P, Wong F, Arroyo V. Diagnosis, prevention and treatment of hepatorenal syndrome in cirrhosis. Gut 2007;56:1310-1318. doi: 10.1136/gut.2006.107789.

[14] McCormick PA, Donnelly C. Management of hepatorenal syndrome. Pharmacol Ther 2008;119:1-6. doi: 10.1016/j.pharmthera.2008.02.012.

[15] Angeli P, Ginès P, Wong F, Bernardi M, Boyer TD, Gerbes A, et al. Diagnosis and management of acute kidney injury in patients with cirrhosis: revised consensus recommendations of the International Club of Ascites. J Hepatol 2015;62:968-974. doi: 10.1016/j.jhep.2014.12.029.

[16] Sarin SK, Kedarisetty CK, Abbas Z, Amarapurkar D, Bihari C, Chan AC, et al. Acute-on-chronic liver failure: consensus recommendations of the Asian Pacific Association for the Study of the Liver (APASL) 2014. Hepatol Int 2014;8:453-471. doi: 10.1007/s12072-014-9580-2.

[17] Mohapatra MK, Behera AK, Karua PC, Bariha PK, Rath A, Aggrawal KC, et al. Urinary bile casts in bile cast nephropathy secondary to severe falciparum malaria. Clin Kidney J 2016;9:644-648. doi: 10.1093/ckj/sfw042. 
Nayak S.L. et al: Bile cast nephropathy in hepatorenal syndrome

[18] Wardle EN. Renal failure in obstructive jaundice-pathogenic factors. Postgrad Med J 1975;51:512-514. doi: 10.1136/pgmj.51.598.512

[19] Betjes MG, Bajema I. The pathology of jaundice-related renal insufficiency: cholemic nephrosis revisited. J Nephrol 2006;19:229-233.

[20] Fickert P, Krones E, Pollheimer MJ, Thueringer A, Moustafa T, Silbert D, et al. Bile acids trigger cholemic nephropathy in common bile-duct-ligated mice. Hepatology 2013;58:2056-2069. doi: 10.1002/hep.26599.

[21] Masumoto T, Masuoka S. Kidney function in the severely jaundiced dog. Am J Surg 1980;140:426-430. doi: 10.1016/0002-9610(80)90183-X

[22] Sant SM, Purandare NM. Cholemic nephrosis-an autopsy and experimental study. J Postgrad Med 1965;11:79-89.

[23] Sanyal AJ, Boyer T, Garcia-Tsao G, Regenstein F, Rossaro L, Appenrodt B et al. A randomized, prospective, double-blind, placebo-controlled trial of terlipressin for type 1 hepatorenal syndrome. Gastroenterology 2008;134: 1360-1368. doi: 10.1053/j.gastro.2008.02.014

[24] Martín-Llahí M, Pépin MN, Guevara M, Díaz F, Torre A, Monescillo A, et al. Terlipressin and albumin vs albumin in patients with cirrhosis and hepatorena syndrome: a randomized study. Gastroenterology 2008;134:1352-1359. doi: $10.1053 /$ j.gastro.2008.02.024.

[25] Gluud LL, Christensen K, Christensen E, Krag A. Systematic review of randomized trials on vasoconstrictor drugs for hepatorenal syndrome. Hepatology 2010;51:576-584. doi: 10.1002/hep.23286.

[26] Heyman SN, Darmon D, Ackerman Z, Rosenberger C, Rosen S. Bile cast nephropathy. Kidney Int 2014;85:479. doi: 10.1038/ki.2013.478.

[27] Jouët $P$, Meyrier A, Mal F, Callard $P$, Guettier C, Stordeur D, et al. Transjugular renal biopsy in the treatment of patients with cirrhosis and renal abnormalities. Hepatology 1996;24:1143-1147. doi: 10.1002/hep.510240527.

[28] Trawalé JM, Paradis V, Rautou PE, Francoz C, Escolano S, Sallée M, et al. The spectrum of renal lesions in patients with cirrhosis: a clinicopathological study. Liver Int 2010;30:725-732. doi: 10.1111/j.1478-3231.2009. 02182.x

[29] Yap DY, Seto WK, Fung J, Chok SH, Chan SC, Chan GC, et al. Serum and urinary biomarkers that predict hepatorenal syndrome in patients with advanced cirrhosis. Dig Liver Dis 2017;49:202-206. doi: 10.1016/j.dld. 2016.11.001. 